\title{
EDITORIAL \\ Clip or coil? Six years of follow-up in BRAT
}

\author{
R. Loch Macdonald, MD, PhD \\ Division of Neurosurgery, St. Michael's Hospital, Labatt Family Centre of Excellence in Brain Injury and Trauma Research, \\ Keenan Research Centre for Biomedical Science, and the Li Ka Shing Knowledge Institute of St. Michael's Hospital, \\ Department of Surgery, University of Toronto, Ontario, Canada
}

A LFRED Blalock, a visionary in surgery, is reported to have said, "It usually requires a considerable time to determine with certainty the virtues of a new method of treatment and usually still longer to ascertain the harmful effects." In neurosurgery, we are confronted with this cautionary view in the consideration of endovascular coiling for ruptured intracranial aneurysms, which is pitted against traditional neurosurgical clipping, at least in cases where there is said to be clinical equipoise. The matter is again raised in this issue of the Journal of Neurosurgery, where the results of the 6-year follow-up in the Barrow Ruptured Aneurysm Trial (BRAT) are published..$^{14}$ Has enough time passed to conclude that coiling is efficacious in some patients? Is another randomized trial needed?

The conclusions of this paper contain a number of post hoc speculations that are certain to incite a flurry of letters and editorials, including this one. The authors should be heartened that this reflects the importance of this data set. Basically, at the 6-year follow-up when the entire data are considered, no difference is observed in poor outcome (that is, a score of $>2$ on the modified Rankin Scale [mRS]) between patients undergoing clipping and patients undergoing coiling. The authors conclude that the study was underpowered to detect a difference between the 2 treatments. They also note that both modalities give the same outcomes for aneurysms of the anterior circulation but that coiling results in better outcomes for those of the posterior circulation. They further observed that coiled aneurysms are less likely to be obliterated and more likely to require retreatment. Obliteration rates and retreatment did not matter much because no rebleeding events occurred, and presumably any complications of retreatment would be reflected in the mRS outcomes. The authors also advocate for another randomized trial.

Let us examine some of these conclusions. BRAT used what has been called a prerandomization strategy, which randomized patients with a subarachnoid hemorrhage (SAH) to clipping or coiling as soon as the SAH was diagnosed. ${ }^{4}$ This randomization approach has advantages and disadvantages, the main disadvantage being inclusion of patients who did not have aneurysms and who thus never had an aneurysm repair. In addition, this strategy resulted in a high crossover rate between the 2 treatment modalities, which is one of the fundamental unfixable issues with BRAT. Randomization was sequential and would therefore be considered pseudorandomization. Various analyses have been published excluding various subsets of these patients. The current analysis is based mainly on "intention to treat according to the assigned treatment group." My understanding of this analysis design is that this means the crossover cases are left in the group to which they were originally assigned, the implications of which I will discuss below. Patients who died before any treatment could be initiated were excluded, but because only 3 patients in each group died, including them in the analysis is not going to materially change the results. Fifty-seven (12\%) of 471 patients did not have aneurysms and were therefore excluded from this analysis. Since these patients' outcomes were the same regardless of which group they were randomized to, including them would only reduce any potential differences between clipping and coiling.

The authors report that 38\% (75 of 199) of patients assigned to coiling crossed over to clipping. This is a high crossover rate and is very problematic because these patients are now not randomized. They crossed over mainly because they had intracerebral hemorrhages, and they did much worse than the other patients. There is no good way to analyze these cases, and arguments that the crossovers favor or penalize coiling could be made. In the analysis, including the crossover patients in the coiled group to which they were randomized will bias against coiling, and 
including them in the clipping group would do the reverse. But this is based only on the outcomes after the crossover patients underwent clipping, and it is not known whether their outcomes would have been different had they undergone coiling. The outcomes with the crossovers excluded may also be biased if, for example, the surgeons performing the coiling had declined to treat these patients in order to make sure the results of the procedures they performed were not affected by patients with a poor prognosis. I do not know of any post hoc randomization analysis that can adjust for this bias caused by the crossovers.

Regarding the lack of statistically significant differences in outcomes between the 2 modalities at the 6-year follow-up, some caveats may be mentioned. While it is commendable that the authors could follow up 336 (82\%) of 408 of patients for 6 years, the loss of $18 \%$ of the patients during the follow-up is a limitation. The International Subarachnoid Aneurysm Trial (ISAT), another trial comparing clipping and coiling, had a five-year or longer follow-up rate of $74 \%$ (1582 of 2143 patients). ${ }^{9}$ In the 6-year BRAT analysis, the last known mRS score was carried forward, but this still resulted in no difference in outcome between clipping and coiling. However, another possible assumption would be to take the worst-case scenario, in which all of the patients lost to follow-up died. I estimate that this would not affect the results, because both groups lost similar numbers of patients during follow-up.

The fundamental issue, however, is the small difference in mRS scores at 1 year in BRAT and also in ISAT. This is taken as evidence that the treatments do not differ much in efficacy. There is another potential explanation, less savory for those performing clipping, which is as follows. The mRS was developed by John Rankin in 1957, who was studying the natural history of 252 patients with cerebral vascular accidents that included a mixture of ischemic strokes, intracerebral hemorrhages, and SAHs. ${ }^{10}$ It is unclear what the actual diagnoses were, and most of the patients affected had focal deficits and probably not an SAH. Good stroke outcomes, as most commonly defined, are those with an mRS score of $0-2$, indicating that patients with a score in this range can look after themselves without assistance. Different questionnaires may be used to assess the mRS score, and they do not necessarily give the same results. The main issue that has been raised is that patients with SAHs and mRS scores of 0-2 often nevertheless have cognitive deficits that prevent them from working and participating in other activities. ${ }^{1}$ It has been reported that coiling is associated with better cognitive outcomes than clipping among patients whose level of dependence is rated as $0-2$ on the mRS. ${ }^{13}$ Thus, it is possible that there might be a big difference in these outcomes between clipping and coiling but that the mRS is insensitive to it.

The next question is: how long should patients be followed up in studies of clipping versus coiling? Whether the ruptured aneurysm is clipped or coiled, a patient is going to die eventually. This outcome is going to be the same for each group, so carrying on with follow-up forever is not useful. In this regard, Table 1 is the key table in the 6-year BRAT study. My interpretation of the data in this table is that at 6 months and at 1 year, outcomes with coiling are better than with clipping and that the results are very similar to those of ISAT. The trend persists after that, but fewer patients are followed up, so the confidence intervals widen, and death from other causes, such as lung cancer and other diseases, creeps in. At the 6-year follow-up in BRAT, poor outcomes were observed in $41 \%$ of patients ( 72 of 174 ) who underwent clipping versus $35 \%$ of patients (57 of 162) undergoing coiling, with an absolute risk reduction (ARR) for coiling of $6 \%$ and a relative risk reduction (RRR) of $17 \%$. At the 5-year follow-up in ISAT, 273 (32\%) patients of 857 who underwent clipping did poorly, versus 241 (28\%) patients of 867 who underwent coiling (with an ARR of $4 \%$ and RRR of $14 \%$ for coiling). ${ }^{9}$ Thus, regardless of statistical significance, the clinical differences are similar between the 2 trials. At the end of the day, coiling extended patients' time with better mRS scores.

The second conclusion was that clipping and coiling resulted in equivalent outcomes among the patients with anterior circulation aneurysms and that those with posterior circulation aneurysms did better with coiling. Aneurysm location, however, was not a prespecified subgroup. The Consolidated Standards of Reporting Trials (CONSORT) guideline suggests that subgroups be prespecified..$^{12}$ The point of this guideline is to disclose a post hoc data analysis that was not driven by a hypothesis generated before the trial began. However, prespecifying a subgroup does not mean the results of that analysis are necessarily true.$^{15}$ Regardless of this lack of prespecification, and as the authors state, BRAT was underpowered to begin with, so subgroup analysis is not going to be adequately powered, either (whatever it is being powered to detect).

The study also contains a detailed analysis of the posterior circulation aneurysm outcomes; this analysis indicated major imbalances in the actual aneurysm repair method that was used after the treatment assignment. It was not clear how many of these imbalances were caused by the crossovers. Are we to conclude that coiling achieves better outcomes for the posterior circulation aneurysms, especially those at the posterior inferior cerebellar artery, for which clipping was worse, even though this is traditionally the "easy" posterior circulation location? And can we assume equivalent outcomes for the basilar bifurcation aneurysms? It is not scientifically possible to reach any conclusions on the basis of this analysis. The numbers of patients are too low, and the crossovers from coiling to clipping are unknown.

The study also presents a detailed analysis of aneurysm obliteration rates and retreatments. These are surrogate outcomes for rebleeding, which is but one factor leading to poor outcomes. Surrogate outcomes have many limitations, and very rigorous criteria have to be met for their acceptance, at least as primary outcomes in clinical trials. ${ }^{6}$ In this study, no rebleeding events were identified, so unfortunately, incomplete aneurysm obliteration does not matter much.

The BRAT study has many strengths. The ability to randomize patients immediately after SAH was demonstrated, and the trial remains only 1 of 3 randomized studies of clipping versus coiling. Several meta-analyses have been performed of the randomized trials of clipping 
versus coiling, ${ }^{7,8}$ and these analyses concluded that coiling was superior to clipping in patients with aneurysms deemed treatable by either method. One meta-analysis was criticized for including BRAT because it is considered a prerandomized trial. ${ }^{5}$ I consider ISAT a good study, but it showed that coiling was better for a select patient population with rather small anterior circulation aneurysms. BRAT is also a key data set, but neither BRAT nor ISAT permit the drawing of scientific conclusions about the benefits of coiling or clipping for posterior circulation aneurysms.

What do we conclude from these latest results? Coiling gave several patients years of better function as measured on the mRS. The authors conclude that the merits of coiling are still debatable and that additional randomized trials are needed. Indeed, Darsaut and colleagues have embarked on such a study. ${ }^{3,11}$ Spetzler and colleagues compare their results with those from ISAT and conclude that if the surgery results in ISAT were as good as they were in BRAT, then clipping would be as good as coiling. I would suggest that it is impossible to make such comparisons between 2 trials like this. Speaking for myself and not for all of the other undoubtedly more skilled surgeons who perform clipping, I am sure that I am not as good at clipping as the authors, and I find it hard to compete against coiling. Advocates of neurosurgical clipping of ruptured aneurysms are fighting an increasingly uphill battle with fewer and fewer troops. Current coiling technology and skills with balloon remodeling and related approaches make it possible to place coils into almost every aneurysm.

Although incomplete aneurysm treatment is common, the authors' review of the literature shows that rebleeding is not as common. As the authors state and we have confirmed, operative complications are a key factor in the difference in outcomes between clipping and coiling. ${ }^{2}$ As fewer neurosurgeons clip aneurysms, it is hard to see how complications are going to go down. While I fully support conducting a trial such as ISAT II, such clinical equipoise for another trial of clipping versus coiling is not widespread in the minds of the burgeoning numbers of those performing endovascular coiling.

http://thejns.org/doi/abs/10.3171/2014.11.JNS142261

\section{References}

1. Al-Khindi T, Macdonald RL, Schweizer TA: Cognitive and functional outcome after aneurysmal subarachnoid hemorrhage. Stroke 41:e519-e536, 2010

2. Ayling OGS, Ibrahim GM, Drake B, Torner JC, Macdonald RL: Operative complications and differences in outcome after clipping and coiling of ruptured intracranial aneurysms. J Neurosurg [epub ahead of print June 5, 2015. DOI: 10.3171/2014.11.JNS141607]

3. Darsaut TE, Jack AS, Kerr RS, Raymond J: International Subarachnoid Aneurysm Trial - ISAT Part II: Study protocol for a randomized controlled trial. Trials 14:156, 2013

4. Darsaut TE, Raymond J: Barrow Ruptured Aneurysm Trial: 3-year results. J Neurosurg 119:1642-1644, 2013

5. Darsaut TE, Raymond J: Rehashing trial results won't help with puzzling aneurysms - patients need best care within a contemporary trial. AJNR Am J Neuroradiol 34:E94-E95, 2013 (Letter)
6. Fleming TR, DeMets DL: Surrogate end points in clinical trials: are we being misled? Ann Intern Med 125:605-613, 1996

7. Lanzino G, Murad MH, D'Urso PI, Rabinstein AA: Coil embolization versus clipping for ruptured intracranial aneurysms: a meta-analysis of prospective controlled published studies. AJNR Am J Neuroradiol 34:1764-1768, 2013

8. Li H, Pan R, Wang H, Rong X, Yin Z, Milgrom DP, et al: Clipping versus coiling for ruptured intracranial aneurysms: a systematic review and meta-analysis. Stroke 44:29-37, 2013

9. Molyneux AJ, Kerr RS, Birks J, Ramzi N, Yarnold J, Sneade $\mathrm{M}$, et al: Risk of recurrent subarachnoid haemorrhage, death, or dependence and standardised mortality ratios after clipping or coiling of an intracranial aneurysm in the International Subarachnoid Aneurysm Trial (ISAT): long-term follow-up. Lancet Neurol 8:427-433, 2009

10. Rankin J: Cerebral vascular accidents in patients over the age of 60. II. Prognosis. Scott Med J 2:200-215, 1957

11. Raymond J, Kotowski M, Darsaut TE, Molyneux AJ, Kerr RS: Ruptured aneurysms and the International Subarachnoid Aneurysm Trial (ISAT): What is known and what remains to be questioned. Neurochirurgie 58:103-114, 2012

12. Schulz KF, Altman DG, Moher D: CONSORT 2010 statement: updated guidelines for reporting parallel group randomised trials. Int J Surg 9:672-677, 2011

13. Scott RB, Eccles F, Molyneux AJ, Kerr RS, Rothwell PM, Carpenter K: Improved cognitive outcomes with endovascular coiling of ruptured intracranial aneurysms: neuropsychological outcomes from the International Subarachnoid Aneurysm Trial (ISAT). Stroke 41:1743-1747, 2010

14. Spetzler RF, McDougall CG, Zabramski JM, Albuquerque FC, Hills NK, Russin JJ, et al: The Barrow Ruptured Aneurysm Trial: 6-year results. J Neurosurg [epub ahead of print June 26, 2015. DOI: 10.3171/2014.9.JNS141749]

15. Wang R, Lagakos SW, Ware JH, Hunter DJ, Drazen JM: Statistics in medicine-reporting of subgroup analyses in clinical trials. N Engl J Med 357:2189-2194, 2007

\section{Response}

\section{Cameron G. McDougall, MD, and Robert F. Spetzler, MD}

Division of Neurological Surgery, Barrow Neurological Institute, St. Joseph's Hospital and Medical Center, Phoenix, Arizona

With pleasure, we read the insightful editorial by Dr. R. Loch Macdonald regarding our most recent results from BRAT. Dr. Macdonald's long experience with clinical trials is clearly apparent in his commentary. He has fairly outlined the strengths and limitations of BRAT, and he gives a well-balanced appraisal of the significance of our results.

In fact, we are in broad agreement with his comments. Respectful of the reader's time, and rather than duplicating or expanding on the details he has stated so well, we will focus more generally on the challenges neurosurgeons must overcome in studying the clip-versus-coil question and in applying the results of such studies.

We face, appropriately so, increasing pressure to base our treatment decisions on high-quality evidence. However, as is common in the surgical world, many decisions must be made in the absence of well-designed trials that apply to the specific situation at hand. This lack of supportive evidence is not for lack of good intentions. Since 
the publication of the ISAT results, ${ }^{2}$ much has been made about their generalizability, and BRAT was intended, in part, to address this issue. But unlike a trial that, for example, compares 2 different medication options, BRAT dealt with a daunting number of confounding variables that are involved when attempting to compare clipping and coiling.

Ideally, a trial comparing the 2 procedures would be conducted in such a way that both procedures are perfectly performed on the appropriate patients at the appropriate time. Each technique would thereby be ideally represented to the extent that any difference between outcomes would reveal the relative limitations of these procedures.

However, this ideal does not exist, so we try instead to make a real-world comparison in hopes of identifying the procedure that generally produces the best results. Although treatment arms may be generally matched, it is clear that there are wide-ranging differences in many interrelated variables (for example, aneurysm location, size, and shape; patient characteristics such as age and clinical condition; and institutional resources and talent) that cannot all be accounted for in a single study. The variables involved in a trial that broadly compares clipping and coiling are so numerous that one would be ill advised to rely on the results of a trial such as BRAT for more than general guidance. Surgical decisions require judgment, and good judgment requires thoughtful consideration of the results of studies, with recognition of the applicabilityor the lack thereof-of study results. Replacing judgment with dogma, using trials selectively to justify biases, and extrapolating trial results beyond what the data support are pitfalls to be avoided.

What can be seen in BRAT and in ISAT is that, while subgroups of patients seem to benefit from coiling, there really are not many differences overall in outcomes between open surgery and aneurysm coiling. With clipping, an increased upfront surgical morbidity rate is offset by a decreased rate of delayed rehemorrhage. Whether this means that one should favor clipping or coiling is somewhat of a value judgment. If the overall difference is ultimately not great, then the implication is that, within a given institution, relatively small differences in any one of the many elements of patient care (for example, practice pattern, judgment, and technical ability) could render irrelevant the results of even the most comprehensive trial.

To address the subgroups more definitively is a formidable task and one that neither BRAT nor ISAT seems well suited to do-BRAT has too few patients and no prescribed subgroup-analysis protocol, and ISAT had too homogenous a cohort of selected aneurysms. Nor does ISAT II $^{1}$ seem well positioned to fill this void any time soon. It may be possible to gain consensus on some subgroup issues from large, well-run cooperative databases and to address specific subsets in well-focused studies when questions persist, but in the near term, many questions remain unanswered. Technological change is relentless, and while the rehemorrhage rate after coiling is decreasing and will continue to decline, a substantial number of ruptured aneurysms are not yet well treated by coiling or by other endovascular techniques.

Developing and promoting the widespread availability of comprehensive care for the patient with a ruptured aneurysm is vitally important. It is fairly common to encounter patients who had suboptimal or partial treatment because a definitive option was not available. It would be most unfortunate for clinical equipoise to be lost solely because open-surgery skills generally fell into decline. Ultimately, despite the inherent challenges, we must make every effort possible to gather the necessary data to allow optimal application of the treatments available. Patients deserve a well-founded answer when they ask, "Which treatment is best?"

\section{Acknowledgments}

We would like to thank Drs. Zabramski, Albuquerque, Hills, Russin, Partovi, Nakaji, and Wallace for their contributions to "The Barrow Ruptured Aneurysm Trial: 6-Year Results (BRAT 6).”

\section{References}

1. Darsaut TE, Jack AS, Kerr RS, Raymond J: International Subarachnoid Aneurysm Trial - ISAT part II: Study protocol for a randomized controlled trial. Trials 14:156, 2013

2. Molyneux A, Kerr R, Stratton I, Sandercock P, Clarke M, Shrimpton J, et al: International Subarachnoid Aneurysm Trial (ISAT) of neurosurgical clipping versus endovascular coiling in 2143 patients with ruptured intracranial aneurysms: a randomised trial. Lancet 360:1267-1274, 2002 